\title{
Dynamic EV Battery Health Recommendations
}

\author{
Markus Eider \\ Deggendorf Institute of Technology \\ Deggendorf, Germany \\ markus.eider@th-deg.de
}

\author{
Andreas Berl \\ Deggendorf Institute of Technology \\ Deggendorf, Germany \\ andreas.berl@th-deg.de
}

\begin{abstract}
Prolonging the lifetime of batteries in Electric Vehicles (EVs) becomes a more and more important issue for private users and fleet operators. In addition to the environmental point of view, a better battery health results in less cost, higher battery capacities and higher performance. To achieve this, the EV drivers or the fleet operators need to get proper information, which kind of actions will increase or decrease the batteries health. To this point, various tips and recommendations exist distributed over literature. Unfortunately, those kind of recommendations are hard to follow in the day-to-day routine. This paper suggests so called dynamic recommendations for battery health that are able to advise the user in specific situations with respect to battery use. Recommendations from literature are broken down into a list, which can be automatically computed. Recommendations will then be dynamically created in the current context of the EV and displayed to the user just in time.
\end{abstract}

\section{CCS CONCEPTS}

- Human-centered computing $\rightarrow \mathrm{HCI}$ theory, concepts and models; Activity centered design; • Applied computing $\rightarrow$ Physics; • Information systems $\rightarrow$ Data analytics;

\section{KEYWORDS}

Electric vehicles, battery health, recommendations, user behaviour

\section{ACM Reference Format:}

Markus Eider and Andreas Berl. 2018. Dynamic EV Battery Health Recommendations. In e-Energy '18: The Ninth International Conference on Future Energy Systems, fune 12-15, 2018, Karlsruhe, Germany. ACM, New York, NY, USA, 7 pages. https://doi.org/10.1145/3208903.3213896

\section{INTRODUCTION}

Batteries make up roughly $50 \%$ of EVs cost $[1,16,23,27]$ and therefore represent a highly valuable part of the EV. Unfortunately, batteries are ageing during usage and time, leading to lower capacity and performance $[2-5,14,16]$. This process is also called degradation. The faster a battery degrades (e.g. due to the usage and charging behaviour of the user), the shorter the lifetime gets and the faster capacity and performance is lowered. EVs users, however expect high performance, high availability, and low costs from their EVs, respectively from their battery.

This work is licensed under a Creative Commons Attribution International 4.0 License.

e-Energy '18, June 12-15, 2018, Karlsruhe, Germany

(c) 2018 Copyright held by the owner/author(s).

ACM ISBN 978-1-4503-5767-8/18/06.

https://doi.org/10.1145/3208903.3213896
In order to prolong battery life and keep battery health on a high level, users should follow general rules, tips, and usage patterns that can be found in literature. These battery usage recommendations comprise the charging of the battery and the usage of the battery, respectively the EVs. In this paper these kind of recommendations are called static recommendations. Users can improve their battery health, e.g., by charging in special ways, driving battery-friendly, choosing suitable routes, and parking their car according to static recommendations. However, it is typically not easy to follow these static recommendations while performing day-to-day tasks with EVs. On one hand static recommendations are written down somewhere in literature, not available during a drive. On the other hand, it is not easy for the EVs user to interpret general recommendations in the current driving or charging context.

This paper suggests so called dynamic recommendations as a solution approach to this problem. Dynamic recommendations are processed from static recommendations, but given to the user just in time and based on the current charging/driving/routing/parking context. Data from the car is gathered and the behaviour of the user is analysed and concrete recommendations are created for the user at specific points of time. In this paper it will be shown that abstract static recommendations from literature can be put into a machine readable table and be processed automatically in order to generate dynamic recommendations. Additionally, two examples of dynamic recommendations will be shown.

The paper is structured as follows: Section 2 discusses background information and static recommendations. Section 3 introduces the concept of dynamic recommendations. Section 4 shows a machine readable representation of recommendations and an algorithm for automatically creating recommendations. Section 5 shows two examples of dynamic recommendations based on real world data. Finally, Section 6 concludes this paper.

\section{BATTERY DEGRADATION AND RECOMMENDATIONS}

In this Section, fundamentals of battery degradation are discussed while considering the battery as a black-box system. In addition, common ageing causes and static recommendations are described in order to derive dynamic recommendations for battery-friendly usage.

\subsection{General Battery Degradation}

Lithium-ion batteries are the one of the most expensive parts in EVs. They make up roughly $50 \%$ of the original vehicle price [27], though their price is dropping due to advancements in the past years (roughly 35\% price drop between 2015 an 2017 [17]). Lithium ion batteries have several benefits, such as their high energy density, 
lifetime, efficiency as well as fast charging properties [8] and are suitable as energy storage systems in EVs [24].

While providing the mentioned benefits, battery performance is impaired by battery degradation through chronological and utilisation causes. For example, Bouchhima et al. [2] explain that the battery health state describe the current EV performance [2]. This health state is influenced by permanent and inevitable degradation $[4,16]$. This is why Li-ion batteries need to be observed and monitored [24] so that the degradation can be limited and safety as well as reliability can be guaranteed [28]. Users of EVs directly influence battery degradation through their utilisation behaviour $[5,25,27]$. Therefore, user behaviours which degrade the battery in an accelerated way need to be identified.

A common metric for the degradation level of a Li-ion battery is the SoH (State of Health) [10] i.a. but it is defined through multiple ways [7]. As and example, the State of Health (SoH) can be computed as the ratio between current and initial capacity [23] i.a. Besides this, manufacturers of EVs and Electronic Control Units (ECUs) keep their calculation approach secret [27]. Thus, it is unknown how the SoH provided by a Battery Management System (BMS) is calculated which makes a health analysis using EV driving data difficult. Hence, a more car-agnostic approach is needed for prolonging battery lifetime in electro mobility.

Batteries degrade in a non-linear way. This is because after the battery health drops below $80 \%$ SoH, the capacity fades rapidly [29]. Thus, the End-of-Life (EOL) of EV batteries is estimated to be $80 \% \mathrm{SoH}$ [2] i.a. The small lifetime range with regards to the $\mathrm{SoH}$ classification therefore needs to be preserved. Hence, dynamic recommendations seem to be a suitable advice for users who want to improve the battery lifetime of their vehicle.

Battery degradation is a permanent as well as dynamical process $[11,21,22]$. It also is an outcome of aggregated battery operation and time, which means that degradation depends on the operation history $[3,5,14]$. The main effects of battery degradation, namely capacity and power fade, can be used to describe degradation but these again do not directly describe the $\mathrm{SoH}$ and can occur independently from each other [8] i.a. However, there are known influences for these main effects which in general cause battery degradation. The most important influences are the ambient temperature, battery current rate, Depth of Discharge (DoD) and the time intervals between full charge cycles [22].

Most studies on battery health and ageing are performed under strict conditions. This is criticised by You et al. [29] since batteries usually degrade under dynamic conditions in practise. Therefore, the practical operation needs to be considered also in order to understand the process of battery degradation. For this reason, EV performance data can be acquired from the EV Controller Area Network (CAN) bus [15, 23, 25], as done in this paper. Another approach, which is more accurate [24], uses a direct measurement of the battery parameters [24] i.a. Though it is difficult to perform [8] as it requires laboratory equipment $[10,24]$ and is therefore not applicable for typical EV users. With the driving data from the EV CAN bus, different driving behaviours can be determined as well [25]. These contain vehicle control actions such as the usage of acceleration or breaking pedals [25]. Hence, behaviour recommendations could be derived from driving data also.

\subsection{Static Recommendations}

Besides the general context of battery degradation, there exist static recommendations with give advice in order to reduce the effects of cyclic and calendaric ageing of Li-ion batteries. On one hand cyclic ageing takes place because charge throughput into and out of the battery during cycles of driving and charging $[2,22,26]$ at typical EV use. On the other, calendaric ageing is observed over the course of time and the powered off state of the EV. It takes place if there is no current flow through the battery [12, 19, 21].

2.2.1 Recommendations to Reduce Cyclic Ageing. Cyclic ageing is primarily induced by current flow and the State of Charge (SoC) levels as well as changes. Therefore, static recommendations which reduce these influences are discussed in the following paragraphs.

Here, the degradation is accelerated by internal temperature rise due to high current or power flow [16] i.a. For example, this strongly occurs during fast charging $[12,18,23]$ which is why generally, fast charging should be avoided. However, it could be identified in a prior paper [6], that fast charging at low temperatures $\left(<17^{\circ} \mathrm{C}\right)$ is battery-friendly. In addition, delayed charging also can prolong battery lifetime [23] and therefore, users should wait between charging and driving and vice versa. Thus, high charge throughput during charging is not harmful in every case.

Besides this, the SoC (State of Charge) level and difference is an important key influence for battery degradation [12, 23]. During charging, it is recommended not to exceed an SoC of $80 \%$ [13] as this is harmful for the battery. Also, the DoD (Depth of Discharge) is often used as measure, which is the difference between $100 \%$ and $\mathrm{SoC}$ at a certain point in time [20]. In general, it should be avoided to frequently discharge the battery to a high $\mathrm{DoD}$ ([2] i.a.) in order to prolong battery lifetime. Furthermore, the SoC difference during charging or discharging should be kept low, as this also accelerates battery degradation $[2,16,18]$.

At EV driving, the utilisation by users has a high influence on battery health. Here, the discharge rate depends on route topology, vehicle load, speed, acceleration and the driving style [1, 22]. Again, this discharge rate influences the level of cyclic ageing. This is summarised in an experiment presented by You et al. [29], where different route types are analysed for their battery health impact [29]. There, capacity fade was highest, if highway drives are performed. A more slightly fade was observed with urban routes and the least capacity fade was determined for city routes. Thus, users should plan their trips on city streets or urban roads.

2.2.2 Recommendations to Reduce Calendaric Ageing. The process of calendaric ageing is independent from cyclic ageing [11, 19], as there is no charge transferred from or to the battery [21]. Since EVs are parked for $80 \%$ to $95 \%$ of their overall lifetime, this degradation type is highly important ([19] i.a.) and recommendations to reduce its effects on battery health must be considered. The following paragraphs summarize the key influences for calendaric ageing, namely the battery temperature as well as SoC levels.

In calendaric ageing, the most influential degradation cause is the ambient temperature [12] i.a. Here, the EV battery should be kept in a range of 15 to $50{ }^{\circ} \mathrm{C}$, as summarized by Rezvanizaniani et al. [22]. In this context, the rate of degradation is doubling with every temperature increase of $10{ }^{\circ} \mathrm{C}$ [22]. Hasan et al. [9] specify 
this range even narrower. They describe that batteries should be operated between 25 and $30{ }^{\circ} \mathrm{C}$ [9]. Extreme temperatures outside of these ranges are considered harmful for battery health [15] i.a.

Similar as in cyclic ageing, the SoC level needs to be considered as ageing influence [2] i.a. On one hand, a high SoC during parking should be avoided since this decreases battery lifetime ([23] i.a.). On the other hand, keeping an EV parked at a low SoC (of 30\% to $40 \%$ ) minimizes capacity fade [11]. Thus, an EV should be parked with its battery being charged to a SoC of $50 \%$ or less [12, 23].

\section{DYNAMIC RECOMMENDATIONS}

This Section provides a definition and the properties for dynamic recommendations. This way, required inputs for generating recommendations can be identified. Besides this, it is discussed which information dynamic recommendations are providing and how users can benefit from them.

Definition [Dynamic recommendation]: A dynamic recommendation in the context of EV battery health is a specific recommendation for driving, parking or charging of the EV. The dynamic recommendation will be pesented to the user right on time when it can be applied. The presented recommendations are based on static recommendations from current state of the art. Recommendations are made dynamic by monitoring an analysing the EV over time in order to select a specific recommendation based on the current state of the EV.

Dynamic recommendations are based on EV data in the past, current situation and in the future. In contrast to this, static recommendations also consider EV use but are independent from EV data. However, it is known from literature that battery health is a consequence of cumulative battery usage $[3,5,14]$ and therefore, dynamic recommendations need to be provided considering the specific environmental and EV internal dynamics in the past and current situation as well as prognostics on parameter change. With this characteristic, dynamic recommendations can tell for how long in the future they should be followed. For example, if a low ambient temperature is predicted within the next month on average, certain recommendations can be given to the user which are valid for this time frame.

The dynamic recommendations are derived from static recommendations in the literature. They inherit dynamic properties in contrast to static recommendation, as they consider specific parameter values, the user decisions and behaviour as well as chronological aspects. This is because of the following reason. Static recommendations are always valid as they are not depending on certain inputs (e.g. a city drive should be preferred instead of taking urban routes [29]). However, a respective recommendation should only be generated if it is applicable to parameter ranges or user behaviour (at charging/driving/parking) at the current moment. In this paper, the behaviour is not taken as input right now but it can also be used if available. This way, users are just in time provided with only the relevant information for battery friendly EV utilisation.

Next, dynamic recommendations should provide a certain priority. Depending on the EV operation and parameter development, multiple recommendations could be provided at once. However, users need advice which recommendation has the most beneficial impact on battery health. Therefore, each recommendation needs to comprise a priority that could be represented by a weight factor. Especially in the case if there are contradicting recommendations, this weight can help to solve these contradictions. For example, at an ambient temperature of $10^{\circ} \mathrm{C}$ over one month, there can be the recommendation to use fast charging [6]. However, if the temperature rises above $17^{\circ} \mathrm{C}$ for one day, slow charging should be preferred [6]. In such case, a contradiction is present and can be solved using weights. This could be realised by coupling the weights to a dedicated weighting function which for example could use the ambient temperature as input. In the example, suggesting slow charging could have a higher priority than fast charging during the month.

Lastly, dynamic recommendations should be consistent over the course of time. This means that provided recommendations should not oscillate within durations of multiple days. Otherwise, dynamic recommendations could be provided in an alternating pattern, showing strong differences or even contradict. A solution to this can be the storage of previously generated recommendations and using these in a feedback loop of the algorithm.

Static recommendations from literature are assumed to prolong battery lifetime in general. Since dynamic recommendations are based on battery-friendly parameter ranges of static recommendations, it is concluded that dynamic recommendations as well prolong the lifetime. However, if a dynamic recommendation is derived from own data analysis of EV operation data, long-term experiments are required in order to verify their correctness. Also, it depends on the willingness and options of the users to follow the recommendations, in order to increase the lifetime of EV batteries. If a user refuses to follow the proposed suggestions or if certain circumstances such as immediate appointments occur, the dynamic recommendations are not performed and battery lifetime is not improved.

\section{COMPUTATION OF DYNAMIC RECOMMENDATIONS}

Based on the related work in Section 2 and the properties of dynamic recommendations in Section 3, preconditions for dynamic recommendations as well as an exemplary processing algorithm are introduced. These are required to generate dynamic recommendations from EV operation and user behaviour data. With this in mind, Section 4.1 provides a table of decisions and recommendations for the algorithm proposed in Section 4.2.

\subsection{Representation of Recommendations}

In the following, preconditions for dynamic recommendations are described. These preconditions are summarised in Table 1 (left side), which are based on static recommendations from literature (see Section 2) as well as assumptions proposed in this paper.

For the dynamic recommendations, restrictions on the data basis, chronological scope and priorities are set in this paper. As battery health depends on cumulative and long-term battery operation $[3,5,14]$, the current situation as well as long-term operation data should be considered. Also a prognosis of the future (e.g. ambient temperature development) should be incorporated when generating dynamic recommendations. For now, only the current data is analysed. As explained in Section 3, dynamic recommendations are 
Table 1: Preconditions and dynamic recommendations

\begin{tabular}{|c|c|c|c|c|c|c|c|c|c|}
\hline $\begin{array}{c}\text { Charge/ } \\
\text { discharge rate } \\
\text { [C] }\end{array}$ & $\begin{array}{l}\text { SoC } \\
{[\%]}\end{array}$ & $\begin{array}{c}\text { Ambient } \\
\text { temperature } \\
{\left[{ }^{\circ} \mathrm{C}\right]}\end{array}$ & $\begin{array}{l}\text { Route } \\
\text { type }\end{array}$ & $\mathrm{N}^{\circ}$ & $\begin{array}{l}\text { Recommen- } \\
\text { dation group }\end{array}$ & Parameter & Unit & $\begin{array}{l}\text { Range/ } \\
\text { options }\end{array}$ & $\begin{array}{l}\text { Literature } \\
\text { references }\end{array}$ \\
\hline cRate $<1$ & - & $a m b T e m p<17$ & - & 1 & Charging & Power & [C] & {$\left[1, \mathrm{P}_{\max }\right]$} & {$[6,22]$} \\
\hline cRate $\geq 1$ & - & ambTemp $\geq 17$ & - & 2 & Charging & Power & [C] & {$[0,1[$} & {$[6,12,18,22,23]$} \\
\hline- & $s o c \geq 80$ & - & - & 3 & Charging & Power & {$[\mathrm{C}]$} & {$[0]$} & {$[11,23]$} \\
\hline - & - & - & - & 4 & Charging & Charging delay & boolean & $\{$ true $\}$ & [23] \\
\hline cRate $\geq 1^{*}$ & - & ambTemp $\geq 17^{*}$ & - & 5 & Driving & Power & [C] & {$[0,1[$} & {$[6,15,16,22]$} \\
\hline- & - & - & routeType == urban & 7 & Routing & Route type & enum & $\{$ city\} & [29] \\
\hline- & - & - & routeType $==$ highway & 8 & Routing & Route type & enum & $\{$ city, urban\} & [29] \\
\hline- & $s o c \geq 50$ & - & - & 9 & Parking & SoC & [\%] & {$[3350[$} & {$[12,23]$} \\
\hline - & - & ambTemp $<15$ & - & 10 & Parking & Location & enum & \{garage\} & [22] \\
\hline - & - & ambTemp $\geq 50$ & - & 10 & Parking & Location & enum & \{garage\} & [22] \\
\hline
\end{tabular}

generated from user behaviour information amongst other data However, there currently is no such information available in order to process this. The user decisions would be required to provide only the relevant advice to users. Therefore, this information is left out as precondition in this paper. In order to consider the decisions without relying on a user input, machine learning or AI may be used. These techniques could learn from the typical EV operation by the user and provide future prognoses. Regarding priorities, these are also not considered right now. However, these could be introduced as weights based on certain parameters such as the ambient temperature.

In left part of Table 1 , the columns 1 up to 4 describe the necessary preconditions for generating dynamic recommendations. Here, numerical values are on one hand derived from literature and on the other hand based on own assumptions (labelled with asterisks). These values are used as references to be compared with current inputs, which are the charge/discharge rate cRate, the SoC soc, the ambient temperature ambTemp and the route type routeType. The latter can be interpreted as an enumeration type.

Furthermore, the recommendations themselves are then listed in the right part of Table 1 with a respective number in the first column, their output information in columns 2 up to 5 , while the involved references from literature are shown in column 8 . It should be mentioned that both the preconditions and recommendations are connected between each lines. The outputs of the recommendations provide information about user behaviour (Recommendation group), the parameter and its unit to be changed and the exact value range it should be held in.

For example, the first charging recommendation is based on a previous paper [6] where it could be identified that using fast charging $(>20 \mathrm{~kW})$ at low ambient temperature $\left(<17^{\circ} \mathrm{C}\right)$ is beneficial for battery health [6]. In the case of the chosen $\mathrm{EV}$, the charging power corresponds to roughly $1 \mathrm{C}$ (1 C resembles to fully charge an EV within one hour). Therefore, the recommendation for choosing fast charging is provided, if the charging rate is above $1 \mathrm{C}$ and the ambient temperature of $17{ }^{\circ} \mathrm{C}$ is undercut. On the contrary, it was shown in the paper that using fast charging at higher ambient temperature leads to accelerated battery degradation. This result is used for the second charging recommendation.

In the following, the assumptions used for Table 1 are described. First, it is known that drawing high power from the battery at high ambient temperature leads to battery degradation (as explained by Dudézert et al. [5] amongst others). However, no specific temperature is specified in related work with regards to formulating recommendations. Therefore, the same temperature limit is assumed for recommendation $\mathrm{N}^{o}$. Besides the temperature, two assumptions are made regarding the SoC. It is known, that frequently high DoD is harmful to battery health ([1] i.a.) and therefore, a limit of one third of the maximum SoC (33\%) was set for recommendation $\mathrm{N}^{o}$ 6. In addition, a recommendation to park the $\mathrm{EV}$ in a garage is provided, since temperatures of 15 to $50{ }^{\circ} \mathrm{C}$ should be avoided [22]. For instance, the garage should provide a temperature of roughly $20{ }^{\circ} \mathrm{C}$ to reduce ageing effects.

Furthermore, there can be recommendations which are based solely on user intent. This is the case with the fourth recommendation which suggests to waiting between driving and charging and vice versa. It is only depending on the input that the user wants to perform a charging process which is currently not available.

\subsection{Processing of Dynamic Recommendations}

The contents of Table 1 can be automatically computed by considering each table entry as a boolean variable (or predicate). Let $\mathrm{A}_{\mathrm{x}}$ (with $x=1 \ldots n$ ) be a predicate or boolean variable. Then, a dynamic recommendation $\mathrm{R}_{\mathrm{y}}$ (with $y=1 \ldots m$ ) can be derived by:

$$
\mathrm{A}_{1} \wedge \mathrm{A}_{2} \wedge \ldots \mathrm{A}_{n} \Rightarrow \mathrm{R}_{\mathrm{y}}
$$

This computation step is used for all recommendations in Table 1 (right side) and shown as short form in Algorithm 1.

There, a procedure GetRecommendations is defined with the input parameters as used in Table 1 (left). In the procedure, a dynamic list recs, compatible to a Recommendation representation, is initialised first which will be filled with recommendations in the procedure. This is done by checking the input parameters for the defined conditions as shown in lines 4, 8, 14 etc. If a condition is satisfied, a respective recommendation will be created and added to the dynamic list recs. In line 38, a recommendation on delaying charging processes is created without any condition, as this currently is independent from any inputs. Finally, the list of created recommendations is provided in line 40.

Due to the connections of preconditions and recommendations in Table 1, there can be multiple recommendations per condition (see lines 9 to 12). This is however since there is no information 


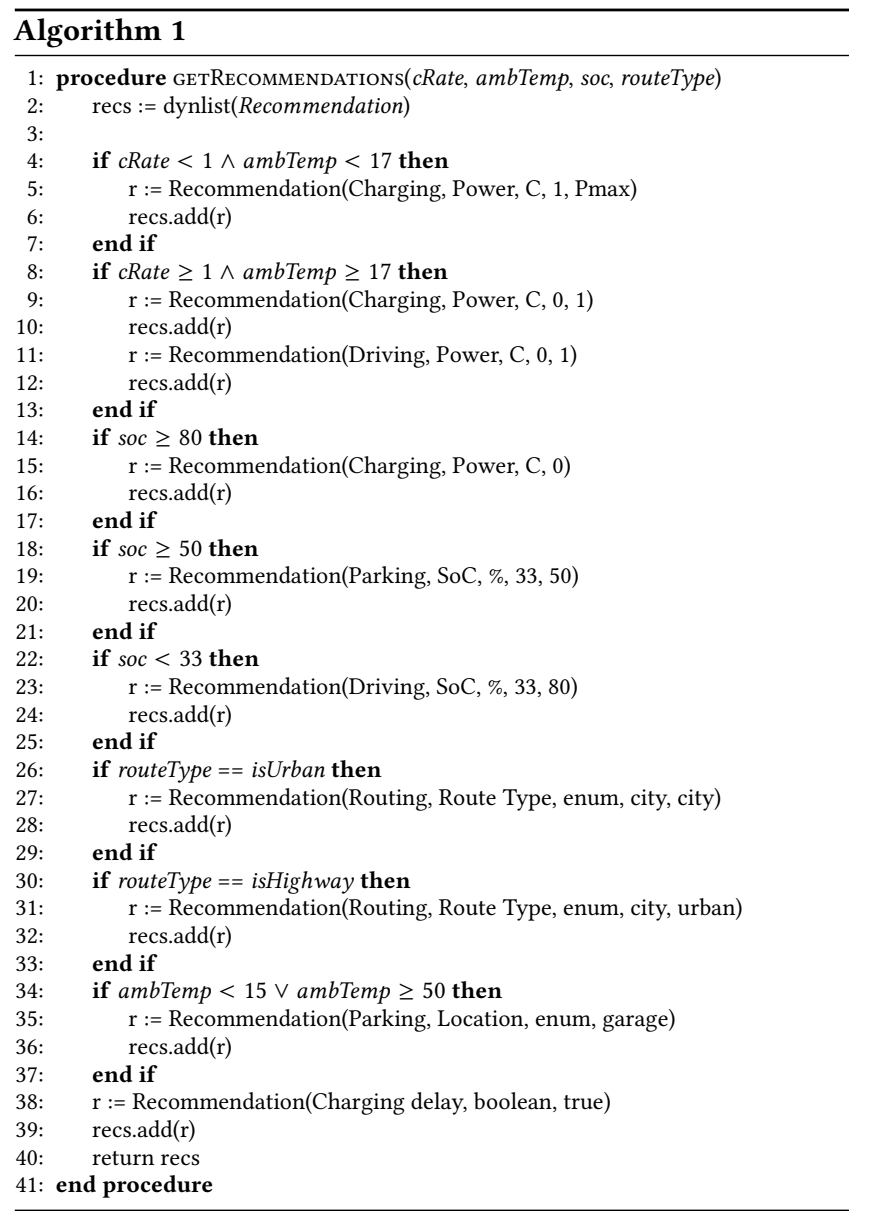

about the user's intent in EV utilisation. Another boolean input would be necessary, which represents if the user wants to initiate a charging process or do a trip in order to split the statement body of the condition in line 8.

\section{EXAMPLES}

In this Section, the priorly discussed processing algorithm is tested on real EV driving data. This is available in the form of historical data acquired from different EVs. In this case, driving data collected from a Nissan Leaf model 2012 is used. Here, the EV is equipped with a data collection system which reads out the CAN bus via a dedicated On-board Diagnostics (OBD) port adaptor. The acquired data is then sent to a database.

The following two Sections show the application of the processing algorithm on data from different trips driven with the EV. The goals of the applications are: (1) to test if the algorithm is working correctly and (2) to demonstrate that not only a single recommendation is generated but multiple recommendations are provided at specific circumstances.

\subsection{Single Recommendation}

This Section describes the test of the decision algorithm for recommendation $\mathrm{N}^{0} 6$ as this recommendations takes only one input, i.e. the SoC. A dynamic recommendation is only produced, if the SoC falls below the threshold of $33 \%$. This allows to see if a recommendation is correctly generated if the condition is satisfied.

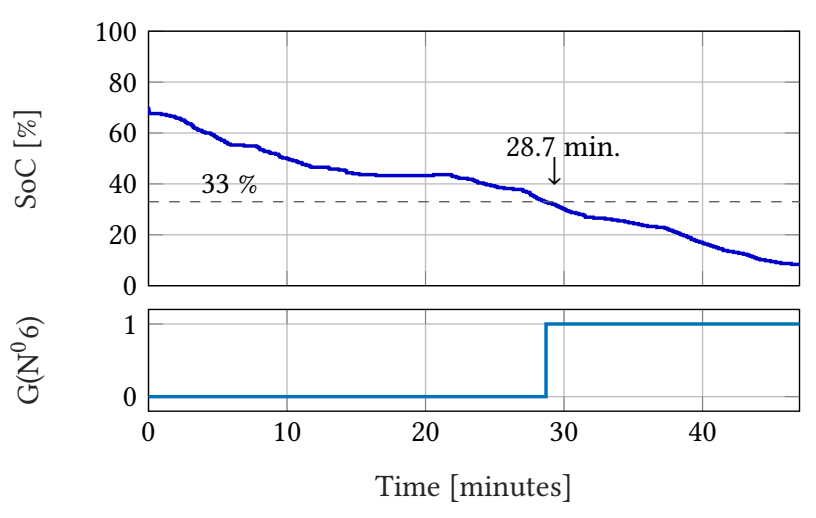

Figure 1: Generation of a single recommendation

For this test, driving data of a single trip from October 2017 is taken. Figure 1 (upper graph) shows the development of the SoC during the trip duration of roughly 47 minutes. Here, the SoC is within a range of $[8.4,70.2] \%$ and shows a nearly steady decline over the trip time frame. Shortly after 20 minutes however, there is a slight increase in SoC which is caused by recuperation of the EV. This means that a height potential loss was converted to electrical energy and stored in the battery while driving downhill.

Until roughly 29 minutes from the trip start, the SoC is above $33 \%$. In this case, the precondition for generating recommendation $\mathrm{N}^{o} 6$ is not satisfied. This is visualised in the bottom graph in Figure 1, where the generator function $\mathrm{G}\left(\mathrm{N}^{o} 6\right)$ describes if the algorithm is providing recommendation $\mathrm{N}^{o} 6$. Hence, $\mathrm{G}\left(\mathrm{N}^{o} 6\right)$ is 0 until 29 minutes. After this time frame, the recommendation is provided and $\mathrm{G}\left(\mathrm{N}^{o} 6\right)$ is 1 .

This test shows, that the proposed algorithm can be applied to real driving data and generates recommendations correctly with regards to the preconditions in Table 1 . However, as there are other recommendations proposed in this paper, further tests are needed. This is discussed in the following Section.

\subsection{Multiple Recommendations}

This Section contains two tests, which visualise that multiple recommendations can be generated simultaneously and that different combinations are possible. Therefore, two trips in Figures 2 and 3 are chosen, where the ambient SoC and ambient temperature $\vartheta_{a m b}$ are within different ranges and exceed the thresholds of the proposed preconditions. These trips were driven also in October 2017, just as in the test of Section 5.1.

In the first test of this Section, the ambient temperature $\vartheta_{\text {amb }}$ moves within $[13.3,15.0]{ }^{\circ} \mathrm{C}$, being at $15^{\circ} \mathrm{C}$ between 4.2 and 10.7 minutes after trip start. The $\mathrm{SoC}$ is within a range of $[61.8,86.2] \%$ and drops below $80 \%$ after 8.4 minutes, as shown in Figure 2 .

As shown in the two bottom graphs in Figure 2, recommendations are generated in specific cases as defined in Table 1. G(N $\left.\mathrm{N}^{o} 3\right)$ is 1 , i.e. recommendation $\mathrm{N}^{o} 3$ is generated, as long as the $\mathrm{SoC}$ is 

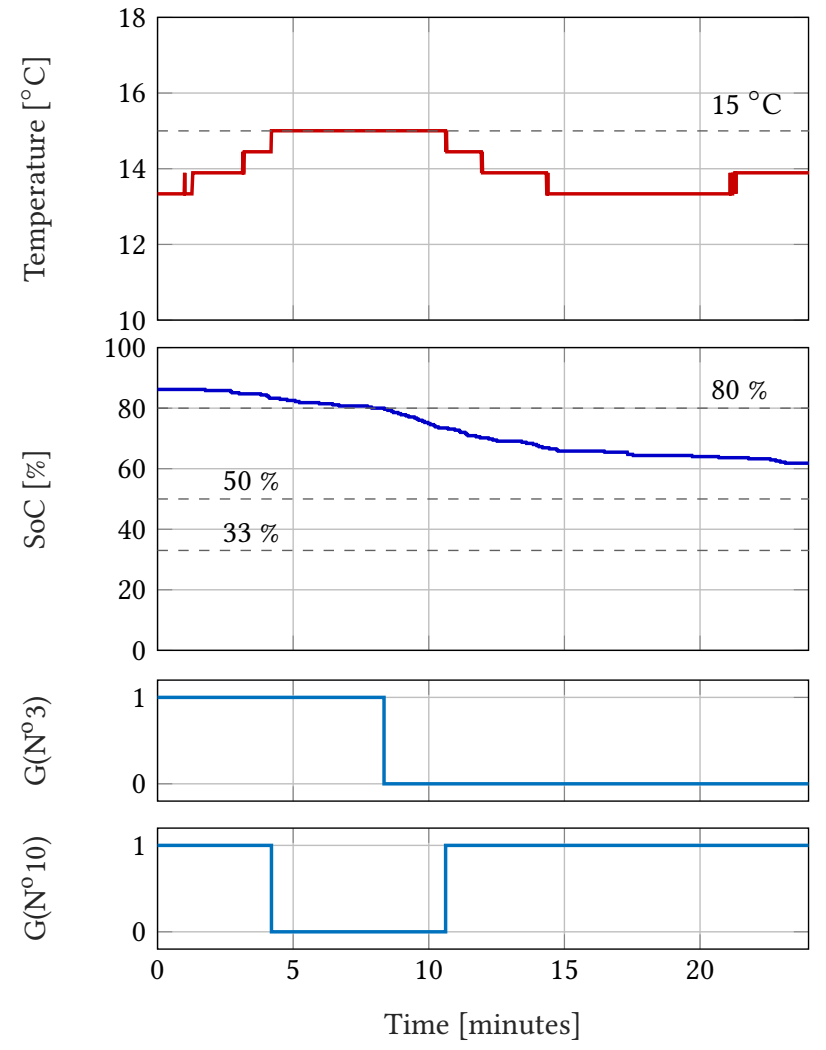

Figure 2: High SoC test with multiple recommendations

above $80 \%$ and therefore satisfies the condition of recommendation $\mathrm{N}^{o} 6$ from the trip start until 8.4 minutes. Also, $\mathrm{G}\left(\mathrm{N}^{o} 10\right)$ is also 1 or 0 according to the recommendation precondition $\left(\vartheta_{a m b} \leq 15{ }^{\circ} \mathrm{C}\right)$.

More interestingly, $\mathrm{G}\left(\mathrm{N}^{o} 6\right)$ never reaches 1 (not shown in Figure 2 ), as the SoC is always above $33 \%$. In addition, recommendations $\mathrm{N}^{o} 4$ and $\mathrm{N}^{o} 9$ are always 1 , as $\mathrm{N}^{o} 4$ does not require any inputs and for $\mathrm{N}^{o} 9$, the SoC has to be at least $50 \%$. Other recommendations $(1,2,5,7,8)$ could not be tested here, as the inputs cRate and routeType are not directly available from the EV CAN bus right now.

In the second test in this Section, the ambient temperature is within $\vartheta_{a m b}=[10.6,16.1]{ }^{\circ} \mathrm{C}$ and the SoC is within the range of $[17.5,47.3] \%$ (see Figure 3). $\vartheta_{a m b}$ drops underneath $15{ }^{\circ} \mathrm{C}$ after 2.4 minutes and SoC $\leq 33 \%$ is satisfied after 2.4 minutes.

As this test describes a different scenario as in the prior test, other combinations of recommendations are provided. Besides the graphs of $\mathrm{G}\left(\mathrm{N}^{o} 6\right)$ and $\mathrm{G}\left(\mathrm{N}^{o} 10\right)$ in Figure $3, \mathrm{G}\left(\mathrm{N}^{o} 4\right)$ is always 1 again, due to the independence of inputs. $\mathrm{G}\left(\mathrm{N}^{o} 3\right)$ and $\mathrm{G}\left(\mathrm{N}^{o} 9\right)$ are always 0 . This is due to the fact that the SoC is never above $50 \%$.

The results visualised in the Figures 2 and 3 as well as the test description show that different combinations and numbers of recommendations can be provided at specific points in time. This fulfils the property of dynamic recommendations that these should be provided at exactly those times when they apply to driving data.

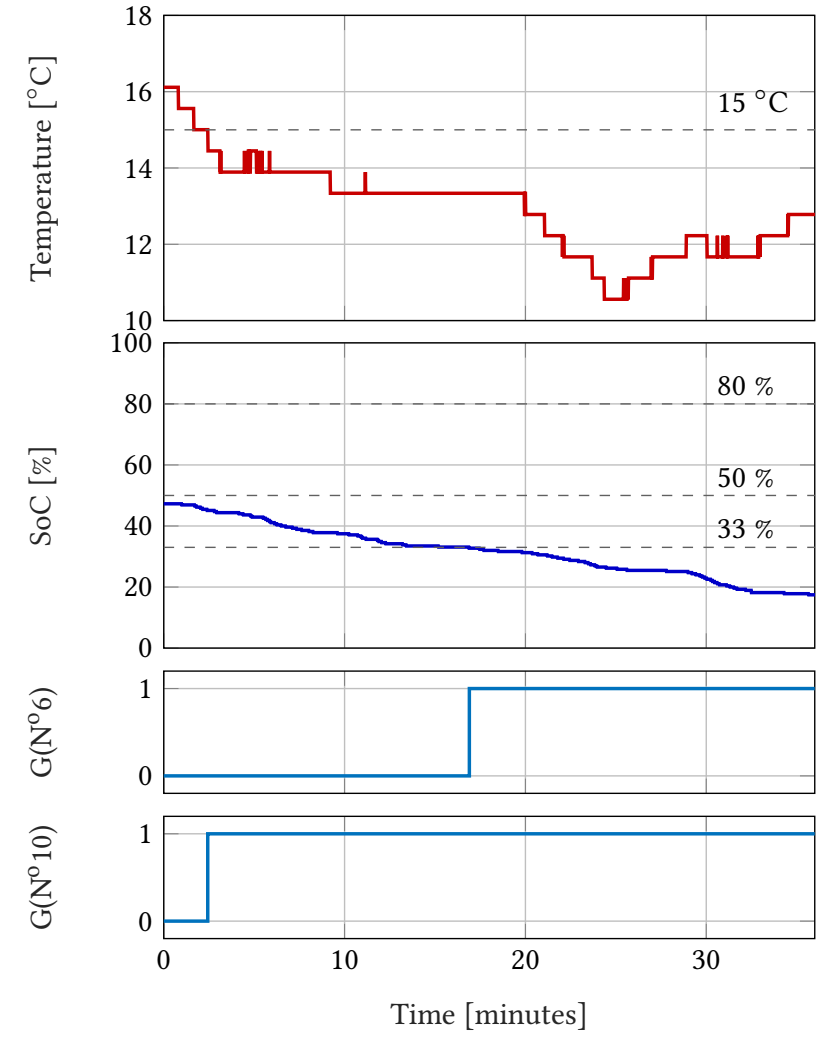

Figure 3: Low SoC test with multiple recommendations

Therefore, the proposed algorithm is suitable as a first solution to implement Table 1 and to process real driving data.

\section{CONCLUSION}

This paper has presented the concept of dynamic recommendations for EV battery health that can be presented to the user just in time in order to improve driving, charging and parking behaviour. The main contributions of this paper are the following:

(1) Dynamic recommendations have been proposed. In contrast to typical battery health tips and recommendations that can be found distributed in literature, dynamic recommendation are presented to the EV user exactly when they can be performed. Dynamic recommendations are able to improve driving, charging and even parking with respect to battery health.

(2) The state of the art of EV battery health recommendations has been broken down into a machine readable table that enables the dynamic generation of recommendations for EV users. Relevant parameters and recommendations have been analysed and groups of recommendations have been created.

(3) A first simple algorithm has been shown in the paper that allows for the automatic interpretation of the recommendation table and can be used to create dynamic recommendations. 
Depending of the current situation of the EV, recommendations can be created just in time to modify the behaviour of the EV user.

(4) Finally, the paper shows two realistic examples of dynamic recommendations. Based on real world data gathered from $\mathrm{EVs}$, input parameters are taken for the control system and dynamic recommendations are created exactly at the point of time, when they are useful to the EV user.

In future work, the dynamic recommendation system for battery health needs to be further improved. On one hand the list of recommendations needs to be extended. On the other hand, the recommendation system needs to be fully developed in order to be able to display recommendations on the EV user's smart phone.

\section{ACKNOWLEDGMENTS}

This research was funded by the European Union Horizon 2020 research and innovation programme under the grant agreement $\mathrm{N}^{\circ} 713864$.

\section{REFERENCES}

[1] Issam Baghdadi, Olivier Briat, Jean-Yves Delétage, Philippe Gyan, and JeanMichel Vinassa. 2016. Lithium battery aging model based on Dakin's degradation approach. Journal of Power Sources 325 (sep 2016), 273-285. https://doi.org/10. 1016/j.jpowsour.2016.06.036

[2] Nejmeddine Bouchhima, Matthias Gossen, Sascha Schulte, and Kai Peter Birke. 2018. Lifetime of self-reconfigurable batteries compared with conventional batteries. Fournal of Energy Storage 15 (feb 2018), 400-407. https://doi.org/10. 1016/j.est.2017.11.014

[3] Chin-Yao Chang, Punit Tulpule, Giorgio Rizzoni, Wei Zhang, and Xinyu Du. 2017 A probabilistic approach for prognosis of battery pack aging. Fournal of Power Sources 347 (apr 2017), 57-68. https://doi.org/10.1016/j.jpowsour.2017.01.130

[4] Hicham Chaoui and Chinemerem Christopher Ibe-Ekeocha. 2017. State of Charge and State of Health Estimation for Lithium Batteries Using Recurrent Neural Networks. IEEE Transactions on Vehicular Technology 66, 10 (oct 2017), 8773-8783. https://doi.org/10.1109/TVT.2017.2715333

[5] C. Dudézert, Y. Reynier, J.-M. Duffault, and S. Franger. 2016. Fatigue damage approach applied to Li-ion batteries ageing characterization. Materials Science and Engineering: B 213 (nov 2016), 177-189. https://doi.org/10.1016/j.mseb.2016. 04.017

[6] Markus Eider, Nicki Bodenschatz, Andreas Berl, Philipp Danner, and Hermann de Meer. 2018. A Novel Approach on Battery Health Monitoring. In Proceed ings of the Iternational Conference of Future Automotive Technologies (CoFAT) Fuerstenfeldbruck, Germany (accepted).

[7] Giuseppe Giordano, Verena Klass, Marten Behm, Goran Lindbergh, and Jonas Sjoberg. 2018. Model-based Lithium-Ion Battery Resistance Estimation from Electric Vehicle Operating Data. IEEE Transactions on Vehicular Technology (2018), 1-1. https://doi.org/10.1109/TVT.2018.2796723

[8] M.A. Hannan, M.S.H. Lipu, A. Hussain, and A. Mohamed. 2017. A review of lithium-ion battery state of charge estimation and management system in electric vehicle applications: Challenges and recommendations. Renewable and Sustainable Energy Reviews 78 (oct 2017), 834-854. https://doi.org/10.1016/j.rser.2017. 05.001

[9] Md. Mehedi Hasan, S. Ali Pourmousavi, Feifei Bai, and Tapan K. Saha. 2017. The impact of temperature on battery degradation for large-scale BESS in PV plant In 2017 Australasian Universities Power Engineering Conference (AUPEC). IEEE, 1-6. https://doi.org/10.1109/AUPEC.2017.8282448

[10] Xiaosong Hu, Jiuchun Jiang, Dongpu Cao, and Bo Egardt. 2015. Battery Health Prognosis for Electric Vehicles Using Sample Entropy and Sparse Bayesian Predictive Modeling. IEEE Transactions on Industrial Electronics (2015), 1-1. https://doi.org/10.1109/TIE.2015.2461523

[11] Peter Keil, Simon F. Schuster, Jörn Wilhelm, Julian Travi, Andreas Hauser, Ralph C Karl, and Andreas Jossen. 2016. Calendar Aging of Lithium-Ion Batteries. Journal of The Electrochemical Society 163, 9 (jul 2016), A1872-A1880. https://doi.org/10. $1149 / 2.0411609$ jes

[12] Gillian Lacey, Tianxiang Jiang, Ghanim Putrus, and Richard Kotter. 2013. The effect of cycling on the state of health of the electric vehicle battery. In 2013 48th International Universities' Power Engineering Conference (UPEC). IEEE, 1-7. https://doi.org/10.1109/UPEC.2013.6715031
[13] Feng Leng, Zhongbao Wei, Cher Ming Tan, and Rachid Yazami. 2017. Hierarchical degradation processes in lithium-ion batteries during ageing. Electrochimica Acta 256 (dec 2017), 52-62. https://doi.org/10.1016/j.electacta.2017.10.007

[14] Yi Li, Mohamed Abdel-Monem, Rahul Gopalakrishnan, Maitane Berecibar, Elise Nanini-Maury, Noshin Omar, Peter van den Bossche, and Joeri Van Mierlo. 2018. A quick on-line state of health estimation method for Li-ion battery with incremental capacity curves processed by Gaussian filter. Fournal of Power Sources 373 (jan 2018), 40-53. https://doi.org/10.1016/j.jpowsour.2017.10.092

[15] Juuso Lindgren and Peter D. Lund. 2016. Effect of extreme temperatures on battery charging and performance of electric vehicles. Fournal of Power Sources 328 (oct 2016), 37-45. https://doi.org/10.1016/j.jpowsour.2016.07.038

[16] Romain Mathieu, Issam Baghdadi, Olivier Briat, Philippe Gyan, and Jean-Michel Vinassa. 2017. D-optimal design of experiments applied to lithium battery for ageing model calibration. Energy 141 (dec 2017), 2108-2119. https://doi.org/10. 1016/j.energy.2017.11.130

[17] George S. Misyris, Antonios Marinopoulos, Dimitrios I. Doukas, Tomas Tengnér, and Dimitris P. Labridis. 2017. On battery state estimation algorithms for electric ship applications. Electric Power Systems Research 151 (oct 2017), 115-124. https: //doi.org/10.1016/j.epsr.2017.05.009

[18] Abdilbari Shifa Mussa, Matilda Klett, Mårten Behm, Göran Lindbergh, and Rakel Wreland Lindström. 2017. Fast-charging to a partial state of charge in lithium-ion batteries: A comparative ageing study. Fournal of Energy Storage 13 (oct 2017), 325-333. https://doi.org/10.1016/j.est.2017.07.004

[19] Jeremy S. Neubauer, Eric Wood, and Ahmad Pesaran. 2015. A Second Life for Electric Vehicle Batteries: Answering Questions on Battery Degradation and Value. SAE International fournal of Materials and Manufacturing 8, 2 (apr 2015), 2015-01-1306. https://doi.org/10.4271/2015-01-1306

[20] Kong Soon Ng, Chin-Sien Moo, Yi-Ping Chen, and Yao-Ching Hsieh. 2009. Enhanced coulomb counting method for estimating state-of-charge and state-ofhealth of lithium-ion batteries. Applied Energy 86, 9 (sep 2009), 1506-1511. https://doi.org/10.1016/j.apenergy.2008.11.021

[21] Eduardo Redondo-Iglesias, Pascal Venet, and Serge Pelissier. 2017. Eyring acceleration model for predicting calendar ageing of lithium-ion batteries. Fournal of Energy Storage 13 (oct 2017), 176-183. https://doi.org/10.1016/j.est.2017.06.009

[22] Seyed Mohammad Rezvanizaniani, Zongchang Liu, Yan Chen, and Jay Lee. 2014. Review and recent advances in battery health monitoring and prognostics technologies for electric vehicle (EV) safety and mobility. Fournal of Power Sources 256 (jun 2014), 110-124. https://doi.org/10.1016/j.jpowsour.2014.01.085

[23] Ivana Semanjski and Sidharta Gautama. 2016. Forecasting the State of Health of Electric Vehicle Batteries to Evaluate the Viability of Car Sharing Practices. Energies 9, 12 (dec 2016), 1025. https://doi.org/10.3390/en9121025

[24] Jinpeng Tian, Rui Xiong, and Quanqing Yu. 2018. Fractional order model based incremental capacity analysis for degradation state recognition of lithium-ion batteries. IEEE Transactions on Industrial Electronics (2018), 1-1. https://doi.org/ 10.1109/TIE.2018.2798606

[25] Chien-Ming Tseng and Chi-Kin Chau. 2017. Personalized Prediction of Vehicle Energy Consumption Based on Participatory Sensing. IEEE Transactions on Intelligent Transportation Systems 18, 11 (nov 2017), 3103-3113. https://doi.org/ 10.1109/TITS.2017.2672880

[26] J. Vetter, P. Novák, M.R. Wagner, C. Veit, K.-C. Möller, J.O. Besenhard, M. Winter, M. Wohlfahrt-Mehrens, C. Vogler, and A. Hammouche. 2005. Ageing mechanisms in lithium-ion batteries. Journal of Power Sources 147, 1-2 (sep 2005), 269-281. https://doi.org/10.1016/j.jpowsour.2005.01.006

[27] Moritz von Hoffen. 2016. Towards an Information System for Evidence-Based Analysis of Charging Behavior, Charging Demand, and Battery Degradation of Electric Vehicles. In 2016 IEEE 18th Conference on Business Informatics (CBI). IEEE, 182-190. https://doi.org/10.1109/CBI.2016.28

[28] Jufeng Yang, Bing Xia, Wenxin Huang, Yuhong Fu, and Chris Mi. 2018. Online state-of-health estimation for lithium-ion batteries using constant-voltage charging current analysis. Applied Energy 212 (feb 2018), 1589-1600. https: //doi.org/10.1016/j.apenergy.2018.01.010

[29] Gae-won You, Sangdo Park, and Dukjin Oh. 2016. Real-time state-of-health estimation for electric vehicle batteries: A data-driven approach. Applied Energy 176 (aug 2016), 92-103. https://doi.org/10.1016/j.apenergy.2016.05.051 